\title{
Two-phase mixed convection nanofluid flow of a dusty tangent hyperbolic past a nonlinearly stretching sheet
}

\author{
A. Mahdy ${ }^{*}$ and G. A. Hoshoudy
}

\section{${ }^{*}$ Correspondence:}

Mahdy:mahdy4@yahoo.com

Mathematics department, Faculty of Science, South Valley University, Qena, Egypt

\begin{abstract}
A theoretical analysis for magnetohydrodynamic (MHD) mixed convection of nonNewtonian tangent hyperbolic nanofluid flow with suspension dust particles along a vertical stretching sheet is carried out. The current model comprises of non-linear partial differential equations expressing conservation of total mass, momentum, and thermal energy for two-phase tangent hyperbolic nanofluid phase and dust particle phase. Primitive similarity formulation is given to mutate the dimensional boundary layer flow field equations into a proper nonlinear ordinary differential system then Runge-Kutta-Fehlberg method (RKF45 method) is applied. Distinct pertinent parameter impact on the fluid or particle velocity, temperature, concentration, and skin friction coefficient is illustrated. Analysis of the obtained computations shows that the flow field is affected appreciably by the existence of suspension dust particles. It is concluded that an increment in the mass concentration of dust particles leads to depreciate the velocity distributions of the nanofluid and dust phases. The numerical computations has been validated with earlier published contributions for a special cases.
\end{abstract}

Keywords: Dusty fluid, Non-newtonian, Tangent hyperbolic, Two-phase nanofluid, Mixed convection

Mathematics Subject Classification (2000): 76A05, 76D05, 76D10, 76E06, 76T15

\section{Introduction}

The flow and heat transfer investigations about non-Newtonian fluids has been of widely importance due to the characteristics of fluid with suspended particles cannot be completely depicted by classical Newtonian fluids theory. Non-Newtonian liquids are widespread fluids in industrial and engineering operations in which linear relationship between stress and deformation rate cannot be obtained. No single non-Newtonian model exists that depicts all the properties of fluids. Tangent hyperbolic fluid model signifies one of interesting non-Newtonian models that developed for chemical engineering systems. This rheological formulation is derived from kinetic theory of liquids rather than the empirical relations. One of the non-Newtonian models introduced by Pop and Ingham [1] was hyperbolic tangent model. Nadeem and Akram[2] has inspected MHD peristaltic flow of a hyperbolic tangent fluid due to a vertical asymmetric channel with heat transfer. Akbar et al. [3] has reported MHD boundary layer flow of non-Newtonian tangent hyperbolic fluid by a stretched sheet numerically. Entropy analysis in MHD steady

(c) The Author(s). 2019 Open Access This article is distributed under the terms of the Creative Commons Attribution 4.0 International License (http://creativecommons.org/licenses/by/4.0/), which permits unrestricted use, distribution, and reproduction in any medium, provided you give appropriate credit to the original author(s) and the source, provide a link to the Creative Commons license, and indicate if changes were made. 
non-Newtonian tangent hyperbolic nanofluid regime through an accelerated stretching cylinder with variable wall temperature has been investigated by Mahdy [4]. Kumar et al. [5] inspected the aspect of partial slip on peristaltic motion of non-Newtonian tangent hyperbolic fluid flow along an inclined cylindrical channel. Naseer et al. [6] have reported the tangent hyperbolic boundary layer fluid flow due to a vertical stretching cylinder. Malik et al. [7] have inspected the MHD flow of non-Newtonian tangent hyperbolic fluid through a stretching cylinder. Hayat et al. [8] have examined the impact of thermal radiation in the two-dimensional free and forced convection flow of a tangent hyperbolic fluid nearby a stagnation point. Some extensive contributions of non-Newtonian fluids have been considered under unlike physical circumstances (Abdul Gaffar et al. [9]; Salahuddin et al. [10]; Nadeem et al. [11]; Mahdy and Chamkha [12], Nadeem and Maraj [13]; Hady et al. [14], Hayat et al. [15]; Mahanthesh et al. [16]).

In higher-power output devices, the forced convection alone is not sufficient in order to dissipate all the heat. As such, combining natural and forced convection (mixed) will usually give desired results. Mainly, the mixed convection phenomenon is found in several industrial and technical applications, for instance, a heat exchanger, nuclear reactors cooling during an emergency shutdown, electronic devices cooled by fans, and solar collectors. Additionally, dusty convective fluid flow has some various applications such as power plant piping, petroleum transport, and wastewater handling and combustion. Gireesha et al. $[17,18]$ have analyzed steady and unsteady MHD boundary layer flow and heat transfer of dusty fluid due to a stretching sheet. Sadia et al. [19] exhibited two-phase free convection flow of nanofluid past a vertical wavy plate. Singleton [20] depicted the boundary layer analysis for dusty fluid. Vajravelu and Nayfeh [21] have explored MHD dusty fluid flow past a stretching sheet. The dynamics of two-phase flow was scrutinized by a number of authors with some various physical conditions (Sivaraj and Kumar [22]; Singh and Singh [23]; Dalal [24]). Flows of mixed convection are of massive interest due to their variety scientific, engineering, and industrial applications in heat and mass transfer. Free and forced convection of mass and heat transfer exist simultaneously in the design fields of chemical operations equipment, distributions of temperature, and formation and dispersion of fog. Also, analysis of flows due to stretched surface through heat transfer has acknowledged ample consideration owing to their possible demands in several industrial procedures, for instance, in metal extrusion, continuous casting, hot rolling, and drawing of plastic films. Particularly in polymer industry, aerodynamic extrusion of plastic sheets has a major significant. This procedure includes the heat transfer between the surrounding and surface fluid. Moreover, the rate of stretching in hot/cold fluids greatly depends upon the quality of the material with desired properties. In such process, heat transfer has an essential role in controlling the cooling rate. Some published investigations can be consulted (Hady et al. [25]; Zeeshan [26]; Khan et al. [27]; Gorla et al. [28]; Mahdy [29-31]; Srinivasacharya and Reddy [32]).

Furthermore, the theory of nanofluids represents an old notion, and it was first presented by Choi and Eastman [33] as they were looking for novel cooling technologies and coolants; thereafter, it became common on account of its extensive applications in heat exchangers, nuclear reactor systems, boilers energy storage, and electronic cooling devices (Ostrach [34]). The ultra-small particle size and thermal conductivity represent the worthy thermophysical properties of nanofluids, and as a result, nanofluids give significantly better performance with comparison to the normal single/multi-phase 
fluids. Makinde [35] analyzed computational modeling of time-dependent nanofluid flow past convectively heated stretched surface. The stagnation point flow of a nanofluid due to unsteady stretching sheet in the presence of slip impacts has been illustrated by Malvandi et al. [36], Mahdy [37], and Mahdy and Sameh [38]. Zheng at al. [39] addressed the behavior of velocity slip in nanofluid flow through a stretching sheet. Also see [40-44].

To the best of the author's knowledge, investigations on mixed convection flow and heat transfer on hydromagnetic dusty non-Newtonian nanofluid flow over a nonlinearly stretching sheet have not been considered so far. In the present analysis, we look at the non-linearly stretching flow of a dusty non-Newtonian tangent hyperbolic nanofluid flow. Numerical computations has been given to illustrate the behavior of nanofluid and dust particles. In our current analysis, we will examine whether the suspension dust particles in nanofluids affects the physical characteristics or not.

\section{Flow field analysis}

A subclass of non-Newtonian fluids is so-called tangent hyperbolic fluid. Actually, in this sort of non-Newtonian fluids [1], the Cauchy stress tensor is given by:

$$
\tau=\left(\mu_{\infty}+\left(\mu_{0}+\mu_{\infty}\right) \tanh (\Gamma \dot{\gamma})^{n}\right)
$$

in which $\tau$ points out extra stress tensor, $\mu_{\infty}$ signifies the infinite shear rate viscosity, $\mu_{0}$ means the zero shear rate viscosity, $\Gamma$ gives the constant of time-dependent material, $n$ refers to the power-law index, i.e., flow behavior index, and $\dot{\gamma}$ is given by the formula

$$
\dot{\gamma}=\sqrt{\frac{1}{2} \sum_{i} \sum_{j} \dot{\gamma}_{i j} \dot{\gamma}_{j i}}=\sqrt{\frac{1}{2} \Pi}
$$

Here, $\Pi=\frac{1}{2} \operatorname{trac}\left(\nabla V+(\nabla V)^{T}\right)^{2}$, and $V$ denotes the velocity vector. We use Eq. (1) for the case as $\mu_{\infty}=0$ since it is not possible to examine the problem for the infinite shear rate viscosity and since we are applying tangent hyperbolic fluid that depicts shear thinning impacts; hence, $\Gamma \dot{\gamma} \ll 1$. Therefor, Eq. (1) mutates to the formula

$$
\begin{aligned}
\tau & =\mu_{0}\left[(\Gamma \dot{\gamma})^{n}\right] \dot{\gamma} \\
& =\mu_{0}\left[(1+\Gamma \dot{\gamma}-1)^{n}\right] \dot{\gamma} \\
& =\mu_{0}[1+n(\Gamma \dot{\gamma}-1)] \dot{\gamma}
\end{aligned}
$$

Now, let us scrutinize a steady, 2-D, incompressible, laminar, boundary layer MHD mixed convection flow of non-Newtonian tangent hyperbolic nanofluid embedded with dust particles towards a vertical nonlinearly stretching sheet. The scenario of a typical flow has been depicted in Fig. 1. As clarified, the orthogonal system $(x, y)$ is chosen, in which the $x$-axis varies along the same stretched sheet direction with stretching rate $U_{w}(x)=a x^{m}$, with $a>0$, and the other axis, i.e., $y$-axis, is being perpendicular to the sheet surface and keeping the origin point to be fixed. An external magnetic field of strength $B_{0}$ is acting in the direction of $y$-axis. As a result to this, the induced current is produced in the fluid and magnetic Reynolds number is treated to be as a small enough such that the induced magnetic field can be ignored.

$T_{\infty}$ gives the fluid temperature far away from the stretching sheet with observation that $T_{w}<T_{\infty}$ and $T_{w}>T_{\infty}$ refers to the opposing and assisting flows, respectively. By following the habitual boundary layer and Boussinesq approximations, the flow-governing 


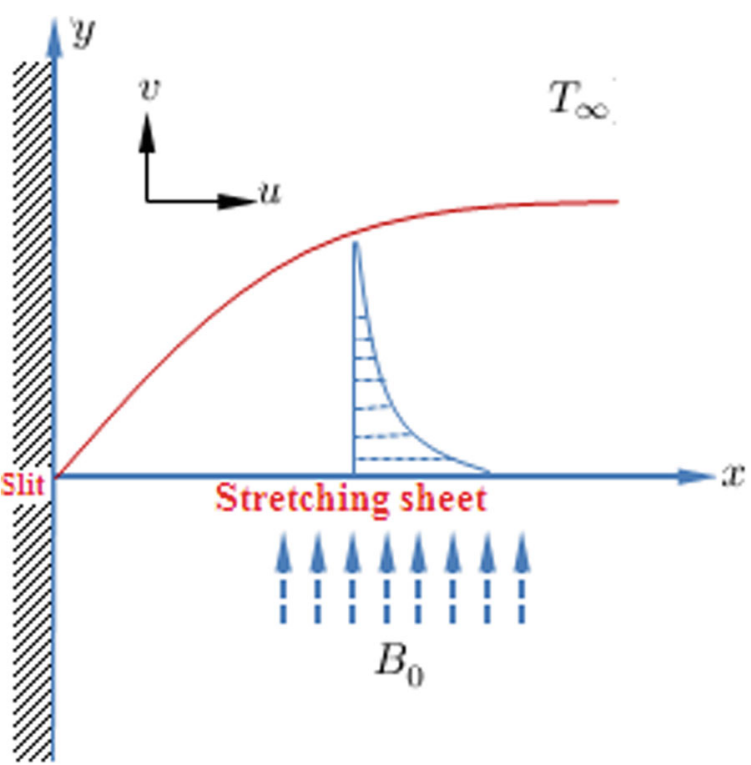

Fig. 1 Orthogonal system and flow model

equations of the steady-state dynamics of a non-Newtonian tangent hyperbolic fluid are given as $[3,17-19,21]$

Fluid phase equations

$$
\begin{aligned}
& \frac{\partial u}{\partial x}+\frac{\partial v}{\partial y}=0 \\
& \begin{aligned}
\rho_{f}\left(u \frac{\partial u}{\partial x}+v \frac{\partial u}{\partial y}\right)= & \mu_{f}\left((1-n)+\sqrt{2} n \Gamma \frac{\partial u}{\partial y}\right) \frac{\partial^{2} u}{\partial y^{2}}+\frac{\rho_{p}}{J}\left(u_{p}-u\right)-\sigma B_{0}^{2} u \\
& +g\left(\left(1-C_{\infty}\right) \rho_{f} \beta\left(T-T_{\infty}\right)-\left(\rho_{n p}-\rho_{f}\right)\left(C-C_{\infty}\right)\right)
\end{aligned} \\
& \begin{aligned}
(\rho c)_{f}\left(u \frac{\partial T}{\partial x}+v \frac{\partial T}{\partial y}\right)=k_{f} \frac{\partial^{2} T}{\partial y^{2}}+(\rho c)_{n p}\left(D_{B} \frac{\partial C}{\partial y} \frac{\partial T}{\partial y}+\left(\frac{D_{T}}{T_{\infty}}\right)\left(\frac{\partial T}{\partial y}\right)^{2}\right) \\
+\frac{\rho_{p} c_{s}}{J^{\star}}\left(T_{p}-T\right)+\frac{\rho_{p}}{J}\left(u_{p}-u\right)^{2}
\end{aligned} \\
& \begin{aligned}
u \frac{\partial C}{\partial x}+v \frac{\partial C}{\partial y}=D_{B} \frac{\partial^{2} C}{\partial y^{2}}+\left(\frac{D_{T}}{T_{\infty}}\right) \frac{\partial^{2} T}{\partial y^{2}} &
\end{aligned}
\end{aligned}
$$

Particle phase equations

$$
\begin{aligned}
& \frac{\partial u_{p}}{\partial x}+\frac{\partial v_{p}}{\partial y}=0 \\
& \rho_{p}\left(u_{p} \frac{\partial u_{p}}{\partial x}+v_{p} \frac{\partial u_{p}}{\partial y}\right)=-\frac{\rho_{p}}{J}\left(u_{p}-u\right) \\
& \rho_{p} c_{s}\left(u_{p} \frac{\partial T_{p}}{\partial x}+v_{p} \frac{\partial T_{p}}{\partial y}\right)=-\frac{\rho_{p} c_{s}}{J^{\star}}\left(T_{p}-T\right)
\end{aligned}
$$

with $(u, v)$ and $\left(u_{p}, v_{p}\right)$ signifying the tangent hyperbolic nanofluid and particle phase velocity components through $x$ and $y$ axes, respectively; $\rho_{f}, \rho_{p}$ give the fluid and dust particle phases density, $\rho_{n p}$ is the density of nanoparticles; $J, J^{\star}$ refer to the dust particles momentum and thermal relaxation time; and $c_{f}, c_{s}$ denote specific heat for the 
fluid and particle phases. $T, T_{p}$ indicate the fluid and dust particle phase temperatures inside the boundary layer; $g$ signifies acceleration due to the gravity; $\Gamma$ indicates the Williamson parameter; $n$ symbolizes the power law index; $D_{B}, D_{T}$ give the Brownian and thermophoresis diffusion coefficients; $C$ points out the resealed nanoparticle volume fraction; $\beta$ means the coefficient of thermal expansion; and $B_{0}, \sigma$ point out the magnetic field strength and electrical conductivity.

The above-stated essential equations must be solved with the appropriate boundary conditions, in order to evaluate the fluid flow fields and the dust particles. Hence, the convenient boundary conditions for our problem are as follows.

Fluid and particle phases boundary conditions are:

$$
\begin{aligned}
& \eta=0, \quad u=U_{w}, \quad v=0, \quad T=T_{w}, \quad D_{B} \frac{\partial C}{\partial y}+D_{T} \frac{\partial T}{\partial y}=0 \\
& \eta \rightarrow \infty, \quad u, u_{p} \rightarrow 0, \quad v_{p}=v \quad T, T_{p} \rightarrow T_{\infty}, \quad C \rightarrow C_{\infty}
\end{aligned}
$$

Now, to mutate the fundamental governing flow field equations and boundary conditions, namely Eqs. (4)-11), the following dimensionless transformation have been given $[12,45]$ :

$$
\begin{aligned}
& \eta=\left(\frac{a(m+1)}{2 v_{f}}\right)^{1 / 2} x^{\frac{m-1}{2}} y, \quad \psi=\left(\frac{2 a v_{f}}{m+1}\right)^{1 / 2} x^{\frac{m+1}{2}} F(\eta), \\
& \psi_{p}=\left(\frac{2 a v_{f}}{m+1}\right)^{1 / 2} x^{\frac{m+1}{2}} S(\eta), \quad \theta\left(\theta_{p}\right)=\frac{T\left(T_{p}\right)-T_{\infty}}{T_{w}-T_{\infty}}, \quad \phi=\frac{C-C_{\infty}}{C_{\infty}}
\end{aligned}
$$

It is evident that Eqs. (4) and (8) are satisfied automatically where the stream functions $\psi$ and $\psi_{p}$ are given as $u=\frac{\partial \psi}{\partial y}, v=-\frac{\partial \psi}{\partial x}$ and $u_{p}=\frac{\partial \psi_{p}}{\partial y}, v_{p}=-\frac{\partial \psi_{p}}{\partial x}$.

Via the similar transformation that clarified in Eq. (12), the boundary layer Eqs. (5)-(11) are re-presented as:

$$
\begin{aligned}
& \frac{m+1}{2}\left(\left(1-n+n W_{e} F^{\prime \prime}\right) F^{\prime \prime \prime}+F F^{\prime \prime}\right)-m F^{2}+\lambda\left(\theta-N_{r} \phi\right) \\
& +D_{p} \alpha_{d}\left(S^{\prime}-F^{\prime}\right)-M_{g} F^{\prime}=0 \\
& \frac{1}{\operatorname{Pr}} \theta^{\prime \prime}+F \theta^{\prime}+N_{b} \theta^{\prime} \phi^{\prime}+N_{t} \theta^{2}+\frac{4 D_{p} \alpha_{d}}{3(m+1) P r}\left(\theta_{p}-\theta\right) \\
& +\frac{2}{m+1} D_{p} E_{c} \alpha_{d}\left(S^{\prime}-F^{\prime}\right)^{2}=0 \\
& \phi^{\prime \prime}+L e F \phi^{\prime}+\frac{N_{t}}{N_{b}} \theta^{\prime \prime}=0 \\
& S S^{\prime \prime}-\frac{2 m}{m+1} S^{\prime 2}-\frac{2 \alpha_{d}}{m+1}\left(S^{\prime}-F^{\prime}\right)=0 \\
& S \theta_{p}^{\prime}-\frac{4 \alpha_{d}}{3(m+1) \gamma P r}\left(\theta_{p}-\theta\right)=0
\end{aligned}
$$

subjected to the following converted fluid and dust particle phase boundary conditions:

$$
\begin{aligned}
& F(0)=0, \quad F^{\prime}(0)=1, \quad \theta(0)=1, \quad N_{b} \phi^{\prime}(0)+N_{t} \theta^{\prime}(0)=0 \\
& F^{\prime}(\infty) \rightarrow 0, \quad \theta(\infty) \rightarrow 0, \quad \phi(\infty) \rightarrow 0 \\
& S(\infty)=F(\infty), \quad S^{\prime}(\infty) \rightarrow 0, \quad \theta_{p}(\infty) \rightarrow 0
\end{aligned}
$$


The resultant parameters are as follows: $W_{e}=\frac{\sqrt{m+1} \Gamma a^{3 / 2} x^{\frac{3 m-1}{2}}}{v_{f}^{1 / 2}}$ signifies Weissenberg number; $M_{g}=\frac{\sigma B_{0}^{2}}{\rho_{f} a x^{m-1}}$ points out magnetic field parameter; $\lambda=\frac{G r}{R e^{2}}$ denotes the mixed convection parameter; $G r=\frac{g \beta\left(1-C_{\infty}\right)\left(T_{w}-T_{\infty}\right) x^{3}}{v_{f}^{2}}$ refers to Grashof number; $R e=\frac{a x^{m+1}}{v_{f}}$ points out Reynolds number, $N_{r}=\frac{\left(\rho_{n p}-\rho_{f}\right) C_{\infty}}{\rho_{f} \beta\left(T_{w}-T_{\infty}\right)\left(1-C_{\infty}\right)}$ gives the buoyancy ratio parameter; $D_{p}=\frac{\rho_{p}}{\rho_{f}}$ refers to relative density; $\alpha_{d}=\frac{1}{j a x^{m-1}}$ means fluid particle interaction parameter; $P r=\frac{\mu_{f} k_{f}}{c_{f}}$ and $L e=\frac{v_{f}}{D_{B}}$ mean Prandtl and Lewis numbers; $N_{b}=\frac{\left(\rho c c_{n p} D_{B} C_{\infty}\right.}{(\rho c)_{f} v_{f}}$, $N_{t}=\frac{(\rho c)_{n p} D_{T}\left(T_{w}-T_{\infty}\right)}{(\rho c)_{f} T_{\infty} v_{f}}$ indicate the Brownian motion and thermophoresis parameters; and $E_{c}=\frac{a^{2} x^{2 m}}{c_{f}\left(T_{w}-T_{\infty}\right)}$ denotes Eckert number. Note $J^{\star}=\frac{3}{2} \gamma_{J} \operatorname{Pr} ; \gamma=\frac{c_{s}}{c_{f}}$ means the specific heat ratio of the mixture. It is significant to state here that for various mixtures, the term of interaction $\gamma$ has values lies between 0.1 and 10.0 Rudinger (1980). It is noticable that for $\alpha_{d}=0$, the flow is purely governed by the mixed convection in the absent of dusty particles (i.e., carrier phase only).

The skin friction factor $C_{f}$, Nusselt number $N u_{x}$, and Sherwood number $S h_{x}$ represent the two major salient quantities of practical interest in the present investigation which are given as follows:

$$
C_{f}=\frac{\tau_{w}}{\frac{1}{2} \rho U_{w}^{2}}, \quad N u=\frac{x q_{w}}{k_{f}\left(T_{w}-T_{\infty}\right)}, \quad S h_{x}=\frac{x q_{m}}{D_{B} C_{\infty}}
$$

The wall shear stress $\tau_{w}$ and surface heat and mass transfer rate per unit area $q_{w}, q_{m}$ :

$$
\tau_{w}=\mu_{f}\left((1-n) \frac{\partial u}{\partial y}+\frac{n \Gamma}{\sqrt{2}}\left(\frac{\partial u}{\partial y}\right)^{2}\right)_{y=0}, q_{w}=-\left.k_{f} \frac{\partial T}{\partial y}\right|_{y=0}, \quad q_{m}=-\left.D_{B} \frac{\partial C}{\partial y}\right|_{y=0}
$$

Therefore, from the similarity transformation which is clarified in Eq. (12), the local skin friction factor and Nusselt and Sherwood numbers in non-dimensional formula are:

$$
\begin{aligned}
\frac{1}{2} R e^{1 / 2} C_{f} & =\sqrt{\frac{m+1}{2}}\left((1-n) F^{\prime \prime}(0)+\frac{1}{2} n W_{e} F^{\prime \prime 2}(0)\right) \\
R e^{-1 / 2} N u_{x} & =-\sqrt{\frac{m+1}{2}} \theta^{\prime}(0) \\
R e^{-1 / 2} S h_{x} & =-\sqrt{\frac{m+1}{2}} \phi^{\prime}(0)
\end{aligned}
$$

\section{Results and discussion}

The present part provides the graphical and tabular results of the influences of different physical governing parameters on representative velocity, temperature of fluid and dusty particle phases, and concentration distribution which are delineated through Figs. 2, 3, 4, 5, 6, 7, 8, 9, 10, and 11 and Tables 1, 2, and 3. The highly non-linear ordinary differential Eqs. (13)-(17) with respect to the boundary condition (18) have been solved numerically via Runge-Kutta-Fehlberg technique with the help of Matlab software. In this method, the boundary value problem have to be mutated into the initial value problem. Additionally, it is pivotal to choose a finite values of $\eta_{\infty}$. In order to appraise the accuracy of the present numerical results, a comparison of heat transfer results is done with the formerly published data (Rana and Bhargava [46] and Mabood et al. [47]) for the case of clean fluid (non suspended dust particles). The comparison is given in Table 1, and an excellent agreement was noticed. Furthermore, Tables 2 and 3 illustrate the impact of 

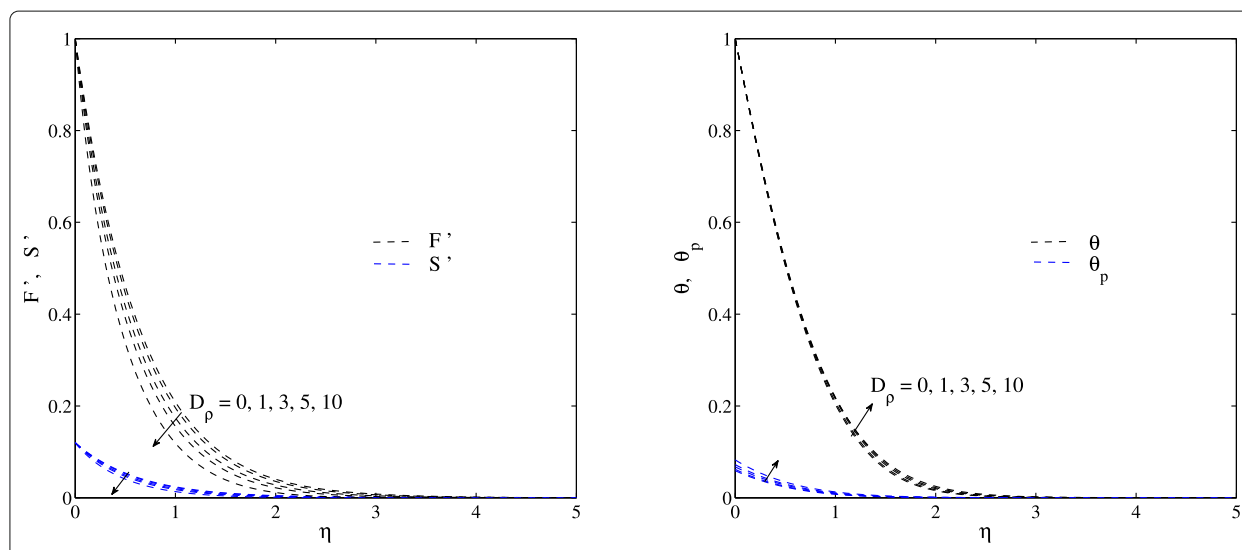

Fig. 2 Velocity and temperature of fluid and dust phase for Dp parameter

governing parameters on skin friction factor and Nusselt and Sherwood numbers. From these two tables, one observation is that the Nusselt number improves with $\gamma, m, N_{b}$, and $\operatorname{Pr}$ while it reduces with the other parameters. In addition, the skin friction factor reduces with $\lambda, E_{c}, m, N_{t}$, and $N_{b}$, whereas it enhances with the other parameters. Here, to illustrate the impact of any physical parameter, the other parameters are considered to be $\operatorname{Pr}=5, \lambda=0.2, W_{e}=0.7, M_{g}=0.5, N_{r}=1, n=0.3, N_{b}=N_{t}=0.4, \alpha_{d}=0.1, D_{\rho}=$ $1, E_{c}=0.3, L e=5, \gamma=0.3$, and $m=0.5$

Figure 2 is prepared to portray the impact of mass concentration of the dust particle parameter $D_{\rho}$ on tangent hyperbolic nanofluid and dust particle phase velocities $F^{\prime}, S^{\prime}$ and temperatures $\theta, \theta_{p}$. An increment in dust particle volume fraction leads to improve the drag force within the fluid, and therefore, velocities are reduced. Again, the aspect of $D_{\rho}$ on nanofluid temperature $\theta$ and dust temprature $\theta_{p}$ is depicted in Fig. 2. With increasing, $D_{\rho}$ maximizes the thermal field of both phases. Theoretically, for larger $D_{\rho}$, more dust particles gain the heat energy from the tangent hyperbolic nanofluid. As a result, cleans fluid's temperature diminished, and relatively the particulate fluid temperature is also diminished.

Figure 3 illustrates the plots for dust particle-fluid interaction parameter $\alpha_{d}$ on the velocity and temperature for nanofluid and dust phases. The tangent hyperbolic nanofluid
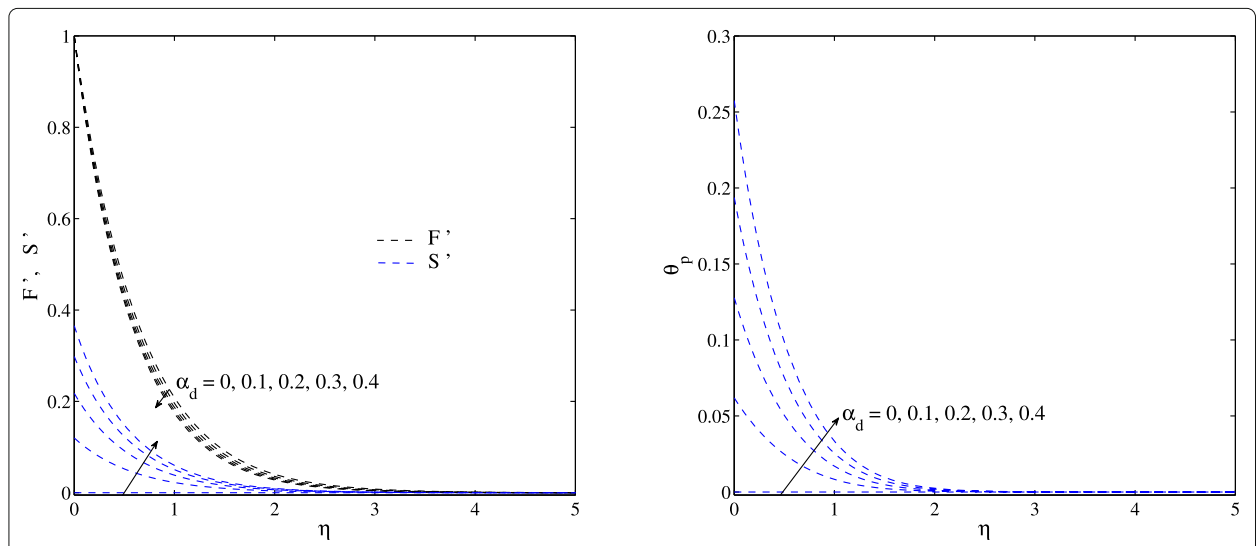

Fig. 3 Velocity and temperature of fluid and dust phase for $\alpha_{d}$ parameter 

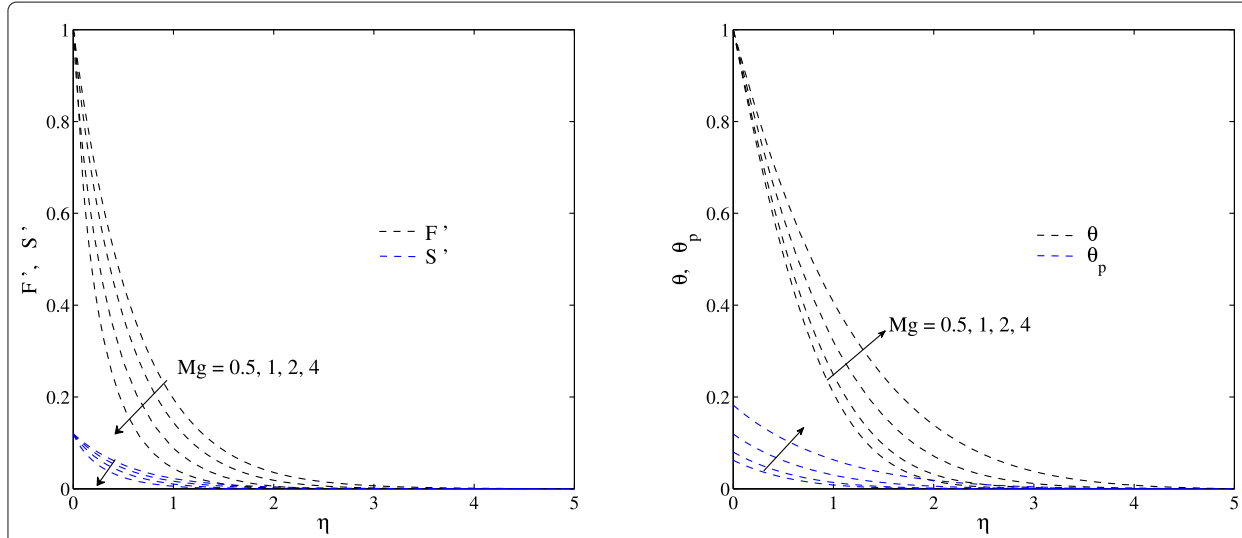

Fig. 4 Velocity and temperature of fluid and dust phase for $\mathrm{Mg}$ parameter
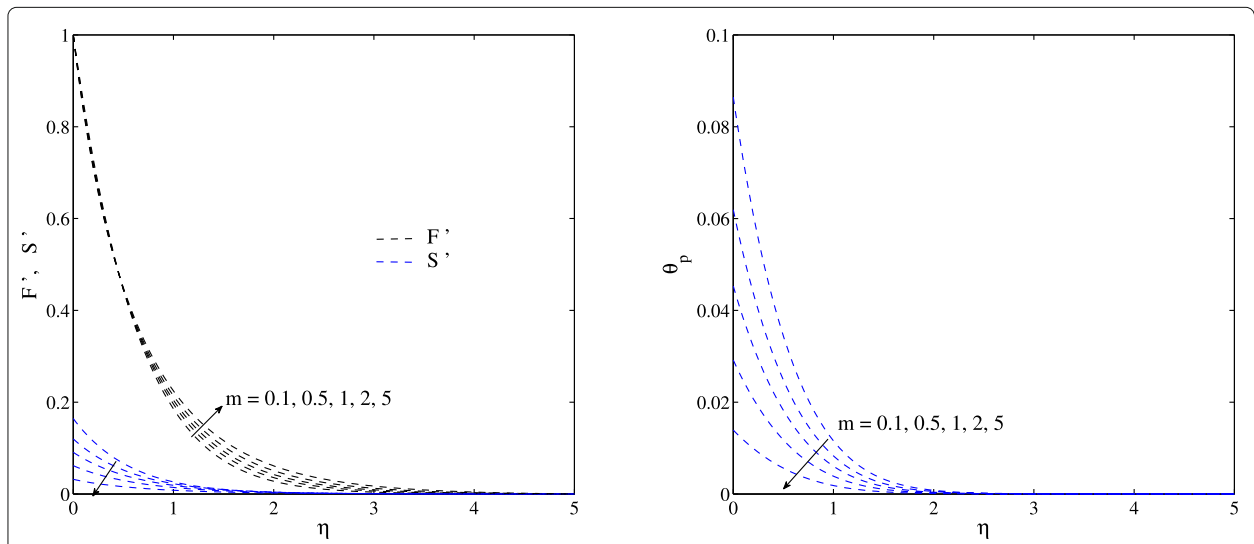

Fig. 5 Velocity and temperature of fluid and dust phase for $m$ parameter
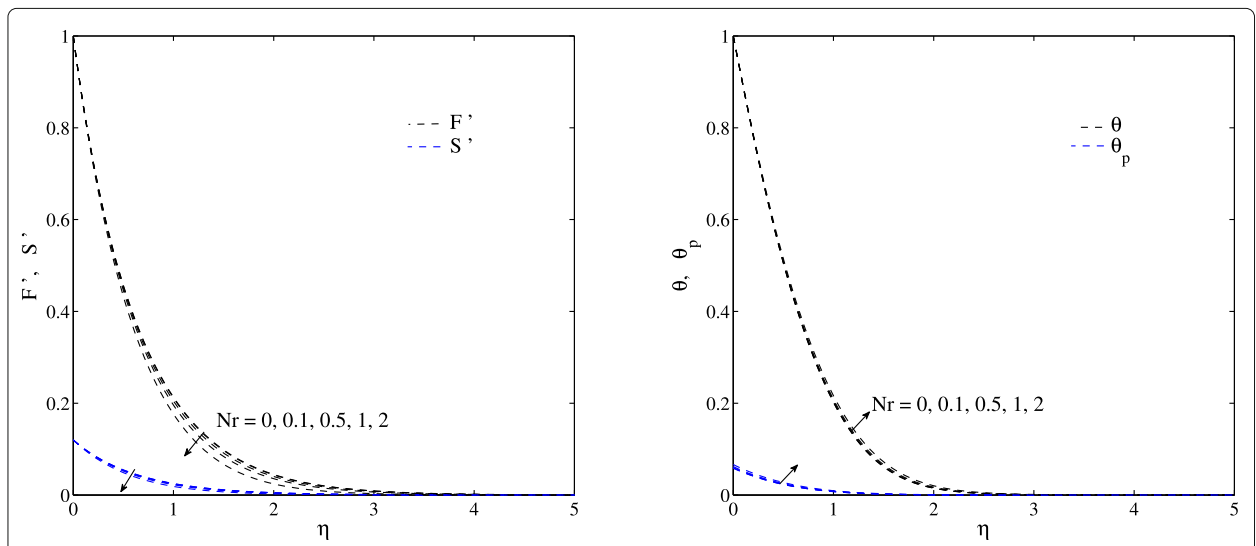

Fig. 6 Velocity and temperature of fluid and dust phase for $\mathrm{Nr}$ parameter 
Mandy and Hoshoudy Journal of the Egyptian Mathematical Society

(2019) 27:44

Page 9 of 16
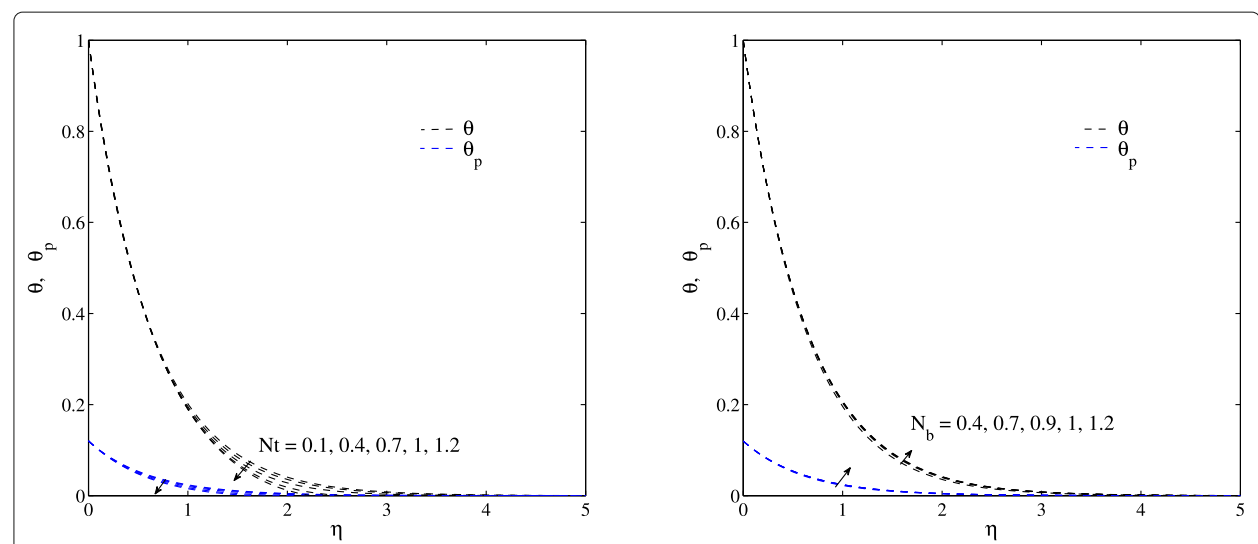

Fig. 7 Fluid and dust temperature phase for $N t, N b$ parameters
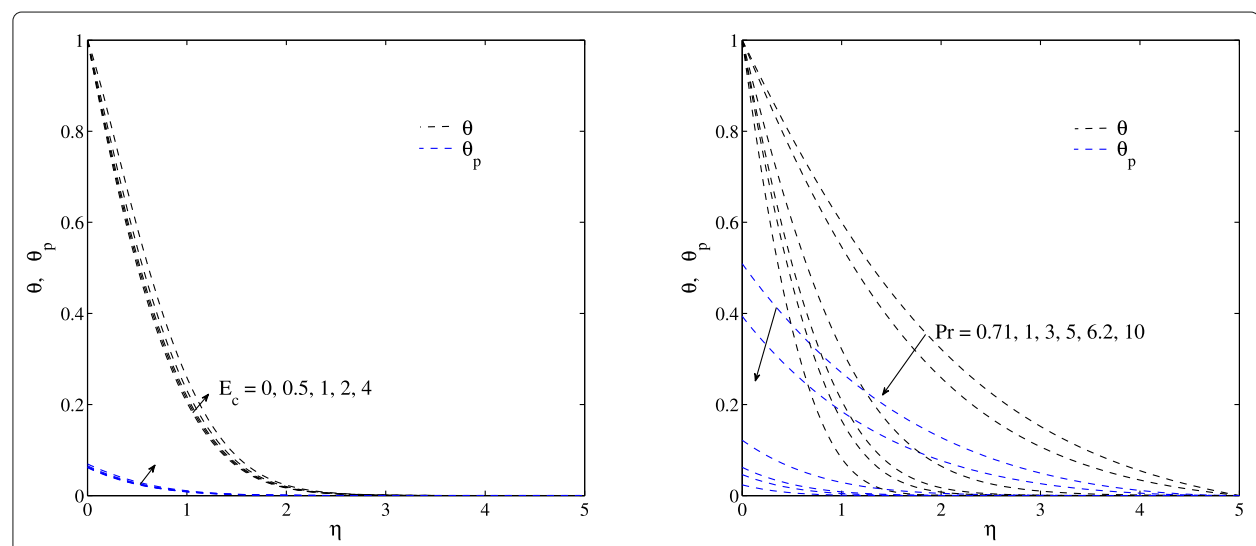

Fig. 8 Concentrations profiles for Nb parameter and Le number
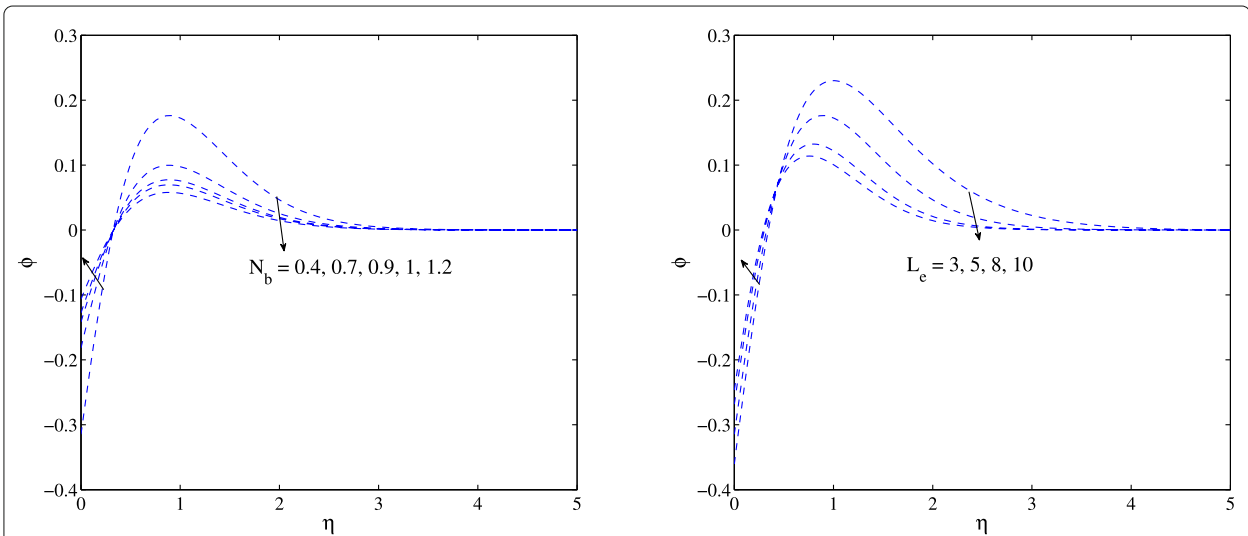

Fig. 9 Fluid and dust temperature phase for $E c$, Pr numbers 

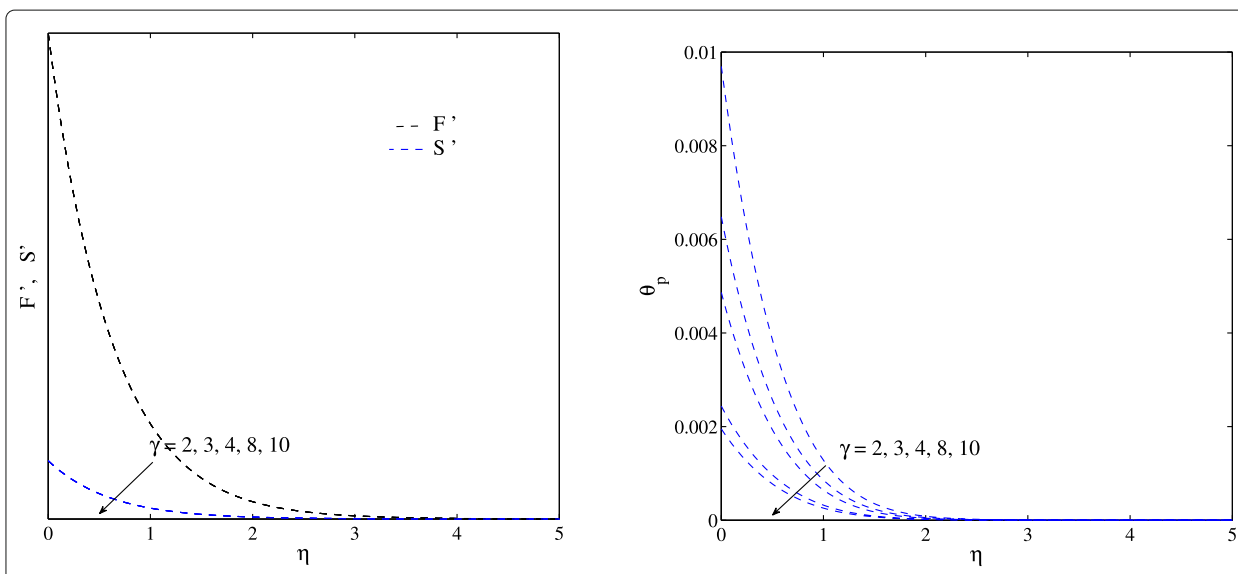

Fig. 10 Velocity and temperature of fluid and dust phase for $\gamma$ parameter

velocity field $F^{\prime}$ is reduced whereas dust particles velocity profile $S^{\prime}$ improved for higher values of $\alpha_{d}$. Physically, a large $\alpha_{d}$ turns to reduce the relaxation time of particle phase and therefore improve the drag force on the fluid in contact with it. It is seen from the figure that, for larger $\alpha_{d}$, the dust particle phase will become in equilibrium with the nanofluid phase. At this point, the temperature and velocity distributions for dust phase become parallel. Truly, due to the interaction with dust particles, the carrier fluid losses the kinetic and thermal energy. Therefore, the carrier nanofluid velocity/temperature field minifies for larger values of $\alpha_{d}$ parameter. This phenomenon indeed occurs for dust particle phase velocity/temperature. The velocity field of accelerated tangent hyperbolic nanofluid, dusty particles, and related momentum boundary layer thicknesses dwindle with increasing of magnetic field parameter $M_{g}$. This can be clarified as the fact that magnetic force acts like resistive force to fluid flow. This means that the applied magnetic field creates a resistive force known as "Lorentz force" which dwindles the fluid motion and results in a thinner momentum boundary layer thickness. The created intensive Lorentz force is accountable for the improvement both of dust temperature $\theta_{p}$ and nanofluid temperature $\theta$ profiles (Fig. 4). For strong magnetic force, this Lorenz force becomes predominant and temperature of fluid rose.
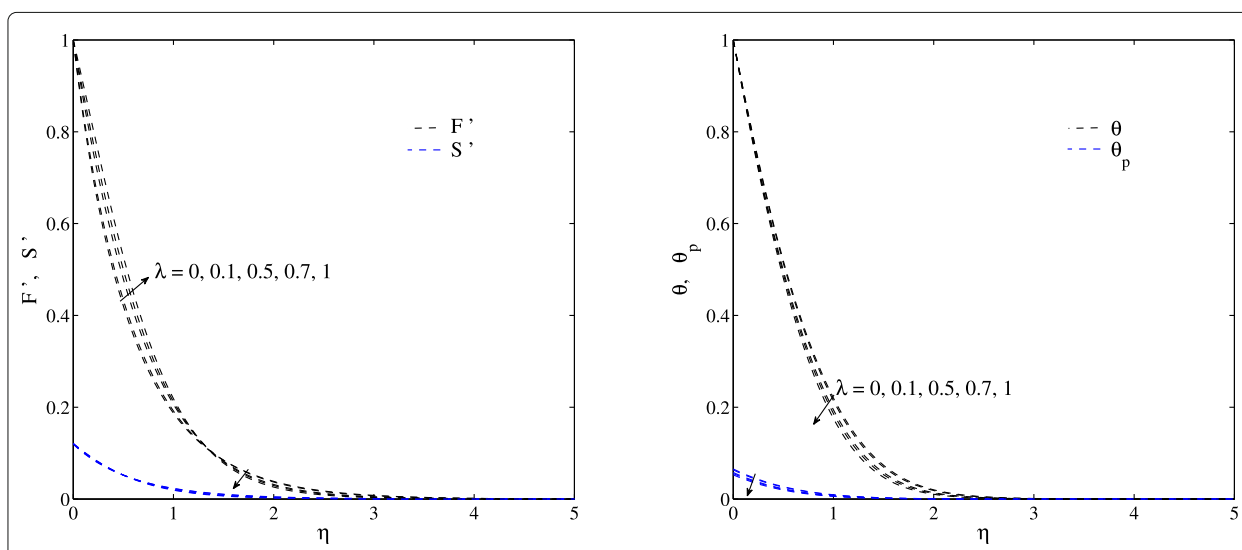

Fig. 11 Velocity and temperature of fluid and dust phase for $\lambda$ parameter 
Figure 5 widths the aspect of nonlinear stretching parameter $m$ on the dimensionless velocity and temperature of nanofluid and dust particle phases. It is observed that the velocity profile of dust particle are insignificantly reduced with increasing values of $m=0.1,0.5,1,2$, and 5 , but the velocity profile of nanofluid is increased. From the same Fig. 5 , it is clear that the variation in the dimensionless dust particle temperature phase due to nonlinear stretching parameter $m$ reduces. Besides, it is seen that an increase in $m$ parameter tends to enhance the temperature gradient at the wall (Table 2). Figure 6 displays the impact of buoyancy ratio $N_{r}$ parameter on the dimensionless velocity and temperature profiles for fluid and dust particle phases. An increment in buoyancy ratio leads to thicken the thermal boundary layer, hence detracting the temperature gradient at the wall, as given in Table 2. Velocity components of nanofluid and dust particle phases reduce with higher values of buoyancy ratio parameter. The temperature profiles of tangent hyperbolic nanofluid and dust particle phases $\theta$ and $\theta_{p}$ for different values of $N_{t}$ and $N_{b}$ are shown in Fig. 7. This figure illustrates that as $N_{t}$ becomes higher, the magnitude of the dimensionless temperatures $\theta$ and $\theta_{p}$ reduces. The diffusion of nanoparticles into the fluid maximizes with the rising in $N_{b}$, and hence, rescaled temperature profiles are improvements (Fig. 7). Impacts of Brownian motion parameter $N_{b}$ and Lewis number Le on concentration distributions are plotted in Fig. 8. The Brownian motion parameter can be illustrated as the ratio of the nanoparticle diffusion, which is due to the Brownian motion impact, to the thermal diffusion in the nanofluid. For the value $N_{b}=0$, thermal transport is absent due to buoyancy impacts produced as a result of nanoparticle concentration gradients. Lewis number $L e$ means the ratio of thermal diffusivity to mass diffusivity. Figure 8 imply that concentration profile is a decreasing function of Lewis number $L e$. Indeed, gradual enlarging values of Lewis number and Brownian motion parameter correspond to minimal mass diffusivity which is accountable in lessening of concentration distribution. Figures 7 and 8 show that the aspects of variations in $N_{b}$ on the variations in concentration profiles are higher than those impacts on the non-dimensional temperature profiles.

The aspect of Eckert number $E_{c}$ for nanofluid and dust temperature distribution were displayed in Fig. 9. From this figure, it is clarified that the nanofluid and dust temperature phases profiles enhance by increasing values of $E_{c}$. It is due to the heat energy is stored in the liquid according to frictional heating and this is true in both cases. Prandtl number $\mathrm{Pr}$
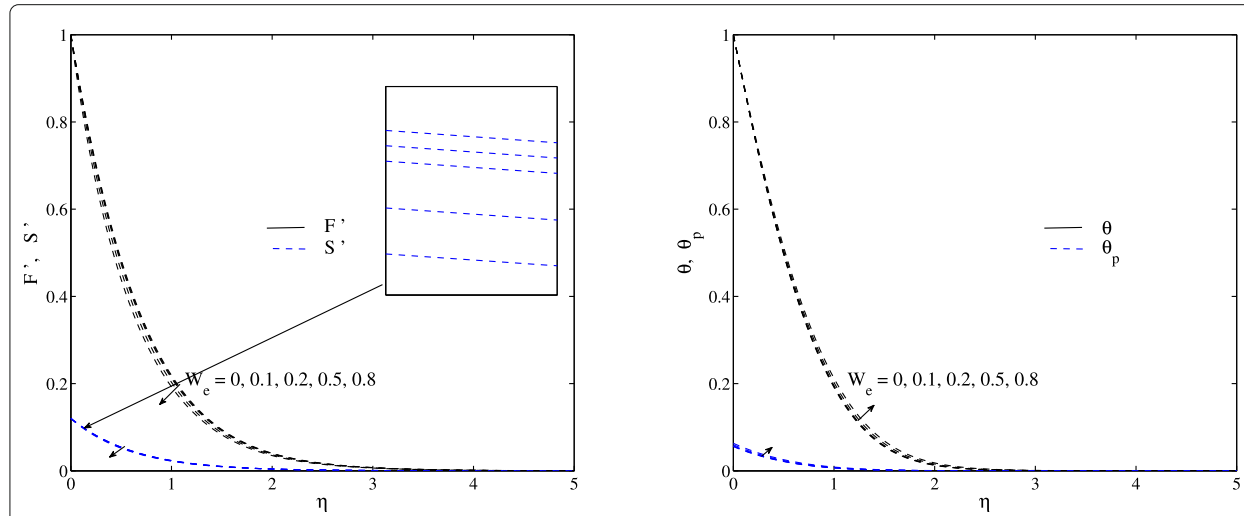

Fig. 12 Velocity and temperature of fluid and dust phase for $W_{e}$ number 

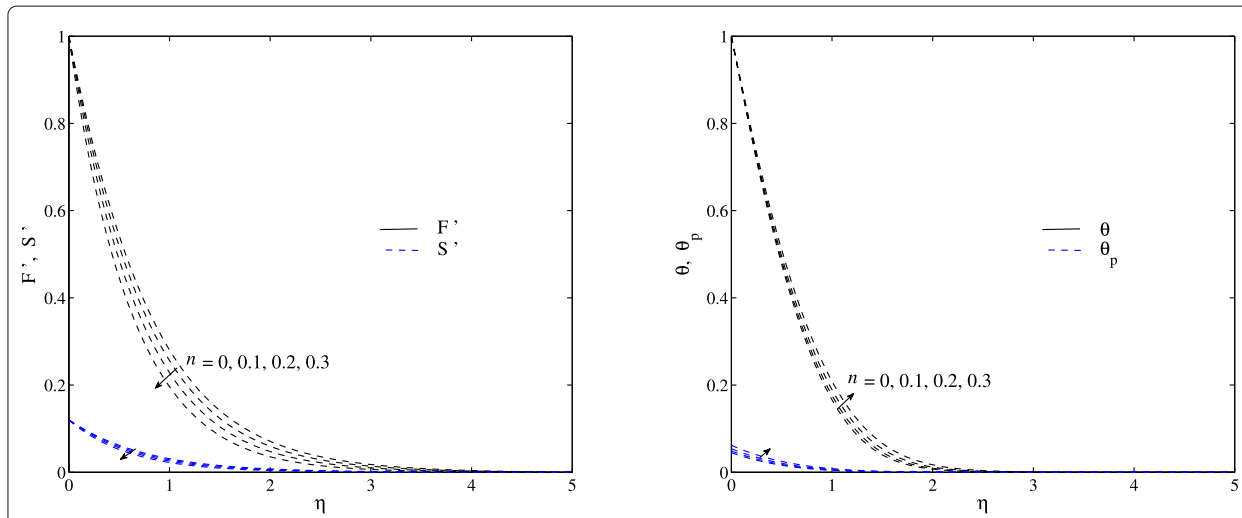

Fig. 13 Velocity and temperature of fluid and dust phase for $n$ parameter

impact on the heat transfer is plotted in Fig. 9. The relative thickening of momentum and thermal boundary layers is governed by Prandtl number. Small values of $\mathrm{Pr}$ will possess higher thermal conductivities, hence heat can diffuse from the sheet very quickly compared to the velocity. Furthermore, it depicts that the temperature reduces with upgrade in the value of Pr. Prandtl number can be used to enhance the rate of cooling. By analyzing the figure, it illustrates that the influence of enlarging $P r$ turns to decrease the nanofluid and dust temperature curves in the flow region, and it is evident that high values of Prandtl number results in thinning of thermal boundary layer. A slight influence has obtained a mixture of specific heat ratio parameter $\gamma$ for velocity and temperature profiles for fluid and dust particle phases in Fig. 10, but $\gamma$ has a strong effect on dust particle temperature distribution. $\theta_{p}$ reduces by increasing $\gamma$.

The impact of mixed convection parameter $\lambda$ on the velocity and temperature curves of nanofluid and dust particle phases is depicted in Fig. 11. The figure reveals that the velocity profiles $F^{\prime}$ and $S^{\prime}$ improve via larger values of mixed convection parameter. The mixed convection parameter presents the ratio of the buoyancy to inertial forces. That is, for higher mixed convection parameter, the buoyancy force overbears the inertial force which enhances the fluid velocity. In addition, the momentum boundary layer thickness improves. Figures 12 and 13 indicate the aspect of the Weissenberg number $W_{e}$ and power law index parameter $n$ on the velocity and temperature profiles for fluid $F^{\prime}$ and dust particles $S^{\prime}$ phases. Both $F^{\prime}$ and $S^{\prime}$ and the associated boundary layer thickness give decreasing

Table 1 Comparison of $-\theta^{\prime}(0)$ with $\operatorname{Pr}=L e=2$ without dust nanoparticles

\begin{tabular}{lllll}
\hline$m$ & $N_{t}$ & Rana and Bhargava [46] & Mabood et al. [47] & Present \\
\hline 0.2 & 0.1 & 0.5160 & 0.5148 & 0.513382 \\
& 0.3 & 0.4533 & 0.4520 & 0.450119 \\
& 0.5 & 0.3999 & 0.3987 & 0.396028 \\
& & & \\
3.0 & 0.1 & 0.4864 & 0.4852 & 0.484279 \\
& 0.3 & 0.4282 & 0.4271 & 0.424702 \\
& 0.5 & 0.3786 & 0.3775 & 0.373428 \\
& & & & \\
10 & 0.1 & 0.4799 & 0.4788 & 0.477941 \\
& 0.3 & 0.4227 & 0.4216 & 0.419096 \\
& 0.5 & 0.3739 & 0.3728 & 0.368370 \\
\hline
\end{tabular}


Table $2 F^{\prime \prime}(0)$ and $-\theta^{\prime}(0)$ for various governing parameters

\begin{tabular}{|c|c|c|c|c|c|c|c|c|c|c|c|}
\hline$\overline{W_{e}}$ & $n$ & $\lambda$ & $\mathrm{Nr}$ & $M_{g}$ & $\rho_{p}$ & $\gamma$ & $\alpha_{d}$ & $E_{c}$ & $m$ & $F^{\prime \prime}(0)$ & $-\theta^{\prime}(0)$ \\
\hline 0.0 & 0.3 & 0.2 & 0.5 & 0.5 & 1 & 0.3 & 0.1 & 0.3 & 0.5 & -1.371359 & 1.140820 \\
\hline 0.1 & & & & & & & & & & -1.397179 & 1.137279 \\
\hline 0.2 & & & & & & & & & & -1.425798 & 1.133512 \\
\hline 0.3 & & & & & & & & & & -1.457876 & 1.129486 \\
\hline \multirow[t]{35}{*}{0.2} & 0.1 & & & & & & & & & -1.229858 & 1.170340 \\
\hline & 0.2 & & & & & & & & & -1.315319 & 1.153947 \\
\hline & 0.3 & & & & & & & & & -1.425798 & 1.133512 \\
\hline & 0.4 & & & & & & & & & -1.578937 & 1.107055 \\
\hline & 0.3 & 0.0 & & & & & & & & -1.566437 & 1.113413 \\
\hline & & 0.1 & & & & & & & & -1.495501 & 1.123730 \\
\hline & & 0.2 & & & & & & & & -1.425798 & 1.133512 \\
\hline & & 0.5 & & & & & & & & -1.223290 & 1.160201 \\
\hline & & 0.2 & 0.0 & & & & & & & -1.424541 & 1.137391 \\
\hline & & & 0.5 & & & & & & & -1.425798 & 1.133512 \\
\hline & & & 0.8 & & & & & & & -1.426856 & 1.131053 \\
\hline & & & 1.0 & & & & & & & -1.427700 & 1.129355 \\
\hline & & & 0.5 & 0.5 & & & & & & -1.425798 & 1.133512 \\
\hline & & & & 1.0 & & & & & & -1.750319 & 1.077091 \\
\hline & & & & 2.0 & & & & & & -2.296064 & 0.987290 \\
\hline & & & & 3.0 & & & & & & -2.763873 & 0.917437 \\
\hline & & & & 0.5 & 0 & & & & & -1.749456 & 1.115311 \\
\hline & & & & & 1 & & & & & -1.800408 & 1.090657 \\
\hline & & & & & 5 & & & & & -2.008776 & 1.008546 \\
\hline & & & & & 10 & & & & & -2.289318 & 0.935892 \\
\hline & & & & & 10 & 2.0 & & & & -2.289318 & 0.935892 \\
\hline & & & & & & 3.0 & & & & -2.289330 & 0.936205 \\
\hline & & & & & & 4.0 & & & & -2.289336 & 0.936361 \\
\hline & & & & & & 8.0 & & & & -2.289344 & 0.936597 \\
\hline & & & & & & 2.0 & 0 & & & -1.749455 & 1.115311 \\
\hline & & & & & & & 0.1 & & & -2.289318 & 0.935892 \\
\hline & & & & & & & 0.2 & & & -2.996140 & 0.914203 \\
\hline & & & & & & & 0.1 & 0.0 & & -2.296627 & 1.232573 \\
\hline & & & & & & & & 0.5 & & -2.289318 & 0.935892 \\
\hline & & & & & & & & 0.8 & & -2.284867 & 0.756204 \\
\hline & & & & & & & & 1.0 & & -2.281871 & 0.635688 \\
\hline & & & & & & & & 0.5 & 0.5 & -2.581250 & 0.908180 \\
\hline & & & & & & & & & 1.0 & -2.438627 & 0.910174 \\
\hline & & & & & & & & & 2.0 & -2.289318 & 0.935892 \\
\hline & & & & & & & & & 5.0 & -2.128768 & 0.996180 \\
\hline
\end{tabular}

behavior for higher values of Weissenberg number $W_{e}$ and power law index parameter $n$ (Fig. 12). In addition, an opposite behavior is shown for temperature of fluid and dust particle phases (Fig. 13).

\section{Conclusions}

This analysis addressed the MHD mixed convection flow of non-Newtonian tangent hyperbolic nanofluid with suspension dust nanoparticles along a nonlinear stretching 
Table $3 F^{\prime \prime}(0),-\theta^{\prime}(0)$, and $-\phi^{\prime}(0)$ for different $N_{t}, N_{b}$ and $\operatorname{Pr}$

\begin{tabular}{llllll}
\hline$N_{t}$ & $N_{b}$ & $P r$ & $F^{\prime \prime}(0)$ & $-\theta^{\prime}(0)$ & $-\phi^{\prime}(0)$ \\
\hline 0.1 & 0.4 & 5.0 & -1.670684 & 1.343005 & -0.335751 \\
0.3 & & -1.656937 & 1.181450 & -0.886087 \\
0.5 & & -1.643514 & 1.030938 & -1.288673 \\
0.7 & & -1.630962 & 0.895652 & -1.567392 \\
0.4 & & -1.650152 & 1.104526 & -1.104526 \\
& 0.4 & -1.648749 & 1.109011 & -0.633720 \\
& 0.7 & & -1.648505 & 1.110275 & -0.493455 \\
& 0.9 & & -1.648436 & 1.110712 & -0.444284 \\
& 1.0 & 0.71 & -1.529700 & 0.440783 & -0.443452 \\
& 0.4 & 3.0 & -1.618851 & 0.864347 & -0.864347 \\
& & -1.650152 & 1.104526 & -1.104526 \\
& 5.0 & -1.688469 & 1.538752 & -1.538752 \\
\hline
\end{tabular}

sheet. Numerical computations are given in tabular and graphical forms in order to clarify the features of nanofluid flow, heat transfer, and dust particle phases. The obtained conclusions are summarized as:

1. Tangent hyperbolic nanofluid phase temperature is greater than the dust particles phase temperature.

2. Both $W_{e}$ and $n$ tend to enhance skin friction factor and reduce rate of heat transfer.

3. Increasing the fluid particle interaction parameter $\alpha_{d}$ leads to reduce the fluid velocity but enhances the velocity and temperature of particle phase.

4. Higher mass concentration of the dust particle parameter gives lower velocities of fluid and dust phases.

5. Nonlinear stretching parameter $m$ has pronounced impact on dust particle phase.

\section{Acknowledgements}

None.

Authors' contributions

All authors jointly worked on the results, and they read and approved the final manuscript.

Funding

Funding information is not applicable/no funding was received.

Availability of data and materials

Not applicable.

\section{Competing interests}

The authors declare that they have no competing interests.

Received: 27 May 2019 Accepted: 18 October 2019

Published online: 07 November 2019

\section{References}

1. Pop, I., Ingham, D. B.: Convective heat transfer: mathematical and computational modeling of viscous fluids and porous media, Pergamon (2001)

2. Nadeem, S., Akram, S.: Magnetohydrodynamic peristaltic flow of a hyperbolic tangent fluid in a vertical asymmetric channel with heat transfer. Acta Mech Sinica. 27, 237-250 (2011)

3. Akbar, N. S., Nadeem, S., Haq, R. U., Khan, Z. H.: Numerical solution of magnetohydrodynamic boundary layer flow of tangent hyperbolic fluid towards a stretching sheet. Indian J. Phys. 87, 1121-1124 (2013)

4. Mahdy, A.: Entropy generation of tangent hyperbolic nanofluid flow past a stretched permeable cylinder: variable wall temperature. Proc IMechE Part E: J Process Mech. Engin. 233, 570-580 (2019)

5. Kumar, Y. V. K. R., Kumar, P. V., Bathul, S.: Effect of slip on peristaltic pumping of a hyperbolic tangent fluid in an inclined asymmetric channel. Adv. Appl. Sci. Res. 5, 91-108 (2014) 
6. Naseer, M., Malik, M. Y., Nadeem, S., Rehman, A.: The boundary layer flow of hyperbolic tangent fluid over a vertical exponentially stretching cylinder. Alex. Eng. J. 53, 747-750 (2014)

7. Malik, M. Y., Salahuddin, T., Hussain, A., Bilal, S.: MHD flow of tangent hyperbolic fluid over a stretching cylinder: using Keller box method. J. Magn. Magn. Mater. 395, 271-276 (2015)

8. Hayat, T., Qayyum, S., Ahmad, B., Waqas, M.: Radiative flow of a tangent hyperbolic fluid with convective conditions and chemical reaction. Eur. Phys. J. Plus. 131(12), 422-443 (2016)

9. Abdul Gaffar, S., Ramachandra, P. V., Anwar, B. O.: Numerical study of flow and heat transfer of non-Newtonian tangent hyperbolic fluid from a sphere with Biot number effects effects. Alex. Eng. J. 54(4), 829-841 (2015)

10. Salahuddin, T., Khan, I., Malik, M. Y., Khan, M., A. Hussain, A., Awais, M.: Internal friction between fluid particles of MHD tangent hyperbolic fluid with heat generation: using coefficients improved by Cash and Karp. Eur. Phys. Plus. J. 132, 205-214 (2017)

11. Nadeem, S., Ashiq, S., Sher Akbar, N., Changhoon, L.: Peristaltic flow of hyperbolic tangent fluid in a diverging tube with heat and mass transfer. Energy. J. Eng. 139(2) (2013)

12. Mahdy, A., Chamkha, A. J.: Unsteady MHD boundary layer flow of tangent hyperbolic two-phase nanofluid of moving stretched porous wedge. Int. Numer. J. Methods Heat Fluid Flow. 28(11), 2567-2580 (2018)

13. Nadeem, S., Maraj, E. N.: The mathematical analysis for peristaltic flow of hyperbolic tangent fluid in a curved channel. Commun. Theor. Phys. 59, 729-736 (2013)

14. Hady, F. M, Mohamed, R. A, Mahdy, A: Non-Darcy natural convection flow along a vertical wavy plate embedded in a n on-Newtonian fluid saturated porous medium. Int. Appl. J. Mech. Eng. 13(1), 91-100 (2008)

15. Hayat, T., Qayyum, S., Alsaedi, A., Shehzad, S. A.: Nonlinear thermal radiation aspects in stagnation point flow of tangent hyperbolic nanofluid with double diffusive convection. Mol. J. Liq. 223, 969-978 (2016)

16. Mahanthesh, B., Sampath, P. B., Kumar Gireesha, B. J., Manjunatha, S., Gorla, R. S. R.: Nonlinear convective and radiated flow of tangent hyperbolic liquid due to stretched surface with convective condition. Results Phys. 7, 2404-2410 (2017)

17. Gireesha, B. J., Manjunatha, S., Bagewadi, C. S.: Unsteady hydromagnetic boundary layer flow and heat transfer of dusty fluid over a stretching sheet. Afrika Metamet. 23(2), 229-241 (2012)

18. Gireesha, B. J, Ramesh, G. K, Subhas, M, Abel Bagewadi, C. S: Boundary layer flow and heat transfer of a dusty fluid flow over a stretching sheet with non-uniform heat source/sink. Int. Multiphase. J. Flow. 37(8), 977-982 (2011)

19. Sadia, S., Nahed, B., Hossain, M. A., Gorla, R. S. R., Abdullah, A. A. A.: Two-phase natural convection dusty nanofluid flow. Int. Heat. J. Mass Transfer. 118, 66-74 (2018)

20. Singleton, R. E.: Fluid mechanics of gas-solid particle flow in boundary layers [Ph.Thesis, D.]. California Institute of Technology (1964)

21. Vajravelu, K., Nayfeh, J.: Hydromagnetic flow of a dusty fluid over a stretching sheet. Int. Nonlinear J. Mech. 27(6), 937-945 (1992)

22. Sivaraj, R., Kumar, B. R.: Unsteady MHD dusty viscoelastic fluid Couette flow in an irregular channel with varying mass diffusion. Int. Heat. J. Mass Transfer. 55, 3076-3089 (2012)

23. Singh, A. K., Singh, N. P.: MHD flow of a dusty visco-elastic liquid through a porous medium between two inclined parallel plates. Proc. Natl. Acad. Sci. India. 66(A), 143-150 (1996)

24. Dalal, D. C., Datta, N., Mukherjea, S. K.: Unsteady natural convection of a dusty fluid in an infinite rectangular channel. Int. Heat. J. Mass Transfer. 41(3), 547-562 (1998)

25. Hady, F. M., Ibrahim, F. S., Abdel-gaied, S. M., Eid, M. R.: Radiation effect on viscous flow of a nanofluid and heat transfer over a nonlinearly stretching sheet. Nanoscale Res. Lett. 7, 299-308 (2012)

26. Zeeshan, A., Majeed, A., Ellahi, R.: Effect of magnetic dipole on viscous ferro-fluid past a stretching surface with thermal radiation. Mol. J. Liq. 215, 549-554 (2016)

27. Khan, S. U., Shehzad, S. A., Rauf, A., Ali, A.: Mixed convection flow of couple stress nanofluid over oscillatory stretching sheet with heat absorption/generation effects. Results Phys. 8, 1223-1231 (2018)

28. Gorla, R. S. R., Lee, J. K., Nakamura, S., Pop, l.: Effects of transverse magnetic field on mixed convection in wall plume of power-law fluids. Int. Eng. J. Sci. 31(7), 1035-1045 (1993)

29. Mahdy, A.: Soret and Dufour effect on double diffusion mixed convection from a vertical surface in a porous medium saturated with a non-Newtonian fluid. Non-Newtonian J. Fluid Mech. 165, 568-575 (2010)

30. Mahdy, A.: Heat transfer and flow of a Casson fluid due to a stretching cylinder with the Soret and Dufour effects. Eng. J. Phys. Thermophys. 88(4), 928-936 (2015)

31. Mahdy Unsteady, A.: MHD slip flow of a non-Newtonian Casson fluid due to stretching sheet with suction or blowing effect. Appl. J. Fluid Mech. 9(2), 785-793 (2016)

32. Srinivasacharya, D., Swamy, G.: Reddy: Mixed convection on a vertical plate in a power-law fluid saturated porous medium with cross diffusion effects. Proc. Eng. 127, 591-597 (2015)

33. Choi, S. U. S., Eastman, J. A.: Enhancing thermal conductivity of fluids with nanoparticles. In: Conference: International mechanical engineering congress and exhibition, San Francisco, CA (United States), 12-17 Nov 1995; Other Information: PBD: Oct, (1995)

34. Ostrach, S.: Natural convection in enclosures. Heat J. Tran. 110, 1175-1190 (1988)

35. Makinde, O. D.: Computational modelling of nanofluids flow over a convectively heated unsteady stretching sheet. Curr. Nanosci. 9, 673-678 (2013)

36. Malvandi, A., Hedayati, F., Ganji, D. D.: Slip effects on unsteady stagnation point flow of a nano fluid over a stretching sheet. Powder Technol. 253, 377-384 (2014)

37. Mahdy, A.: Unsteady mixed convection boundary layer flow and heat transfer of nanofluids due to stretching sheet. Nucl. Eng. Des. 249, 248-255 (2012)

38. Mahdy, A., Sameh, E. A.: Laminar free convection over a vertical wavy surface embedded in a porous medium saturated with a nanofluid. Transp. Porous Med. 91, 423-435 (2012)

39. Zheng, L., Zhang, C., Zhang, X., Zhang, J.: Flow and radiation heat transfer of a nanofluid over a stretching sheet with velocity slip and temperature jump in porous medium. Frankl. J. Inst. 350, 990-1007 (2013)

40. Prakash, J., Sivab, E. P., Tripathi, D., Kothandapani, M.: Nanofluids flow driven by peristaltic pumping in occurrence of magnetohydrodynamics and thermal radiation. Mater. Sci. Semicond. Proc. 100, 290-300 (2019) 
41. Prakash, J., Siva, E. P., Tripathi, D., Kuharat, S., Anwar, O.: Beg: Peristaltic pumping of magnetic nanofluids with thermal radiation and temperature-dependent viscosity effects: modelling a solar magneto-biomimetic nanopump. Renew. Energy. 133, 1308-1326 (2019)

42. Prakash, J., Ravinder, J., Dharmendra, T., Martin, N. A.: Electroosmotic flow of pseudoplastic nanoliquids via peristaltic pumping. J. Braz. Soc. Mech. Sci. Eng. 41, 61-78 (2019)

43. Akbar, N. S., Huda, A. B., Habib, M. B., Tripathi, D.: Nanoparticles shape effects on peristaltic transport of nanofluids in presence of magnetohydrodynamics. Microsyst. Technol. 25, 283-294 (2019)

44. Prakash, J., Ansu, A. K., Tripathi, D.: Alterations in peristaltic pumping of Jeffery nanoliquids with electric and magnetic fields. Meccanica. 53, 3719-3738 (2018)

45. Mahdy, A.: Boundary layer slip flow on diffusion of chemically reactive species over a vertical non-linearity stretching sheet. J. Comput. Theoret. Nanosci. 10(11), 2782-2788 (2013)

46. Rana, P., Bhargava, R.: Flow and heat transfer of a nanofluid over an onlinearly stretching sheet: a numerical study. Commun. Nonlinear Sci. Numer. Simul. 17, 212-226 (2012)

47. Mabood, F., Khan, W. A., Ismail, A. I. M.: MHD boundary layer flow and heat transfer of nanofluids over a nonlinear stretching sheet: A numerical study. Magn. J. Magn. Mater. 37, 4569-4576 (2015)

\section{Publisher's Note}

Springer Nature remains neutral with regard to jurisdictional claims in published maps and institutional affiliations.

\section{Submit your manuscript to a SpringerOpen ${ }^{\circ}$ journal and benefit from:}

- Convenient online submission

- Rigorous peer review

Open access: articles freely available online

High visibility within the field

- Retaining the copyright to your article

Submit your next manuscript at $\gg$ springeropen.com 